\title{
Analysis of blunt injury abdomen. Cases admitted in trauma ward, grh, madurai.
}

\author{
${ }^{1}$ Dr.G.Saravanakumar M.S.,D.A, ${ }^{2}$ Dr.S.Babu. M.S \\ ${ }^{1}$ (Assitant Professor, Department Of General Surgery, Madurai Medical College) \\ ${ }^{2}$ (Associate Professor,Department Of General Surgery,Vellammal Medical College And Hospital,Madurai)
}

Aim:

$>$ Describe the patterns of traumatic abdominal hollow viscus injuries.

$>$ Identify the organs in specific forms of injury.

$>$ Attempt to link the morbidity and mortality to the delay in diagnosis and surgery.

$>$ Detail on the deceptive presentations in blunt injury abdomen.

$\rightarrow$ Produce protocol for the management of hollow viscus injury following blunt injuries in the abdomen.

Materials And Methods: I have analyzed fifty cases of blunt abdominal trauma following road traffic accidents, accidental falls from height and assault by various objects admitted in trauma ward during the period of December 2011 to October 2013

Keywords: Blunt Injury Abdomen, Hollow Viscus Injury, Traumatic Peritonitis

\section{Results}

$>$ The age incidence of blunt injury is highest for the age group 30-39 years which constitutes $28 \%$ of the study population.

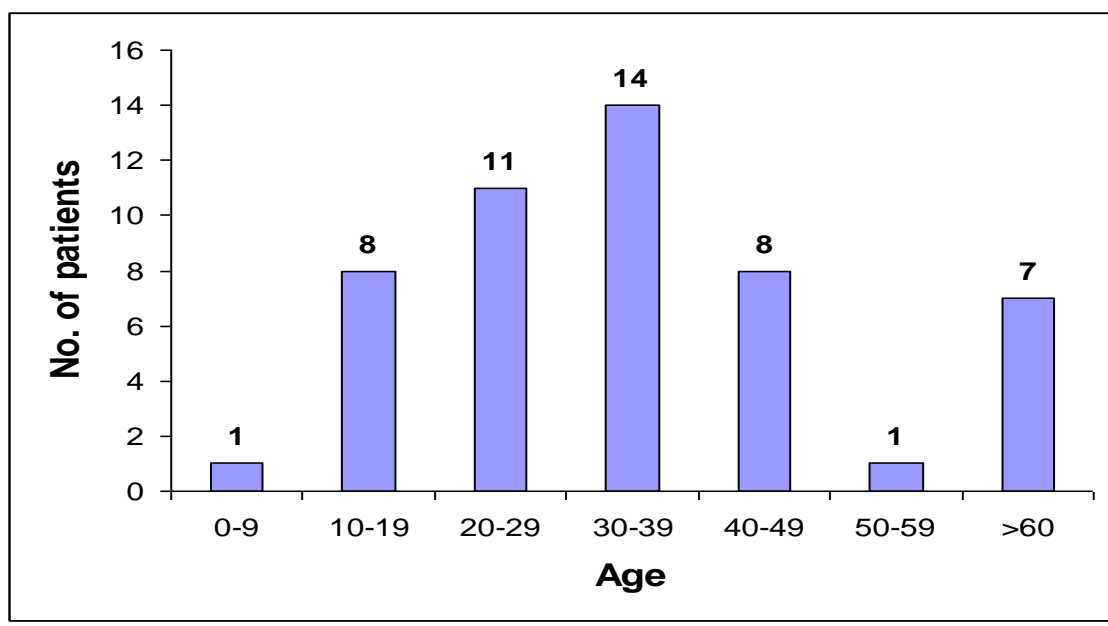

Nature of blunt injury abdomen in our study
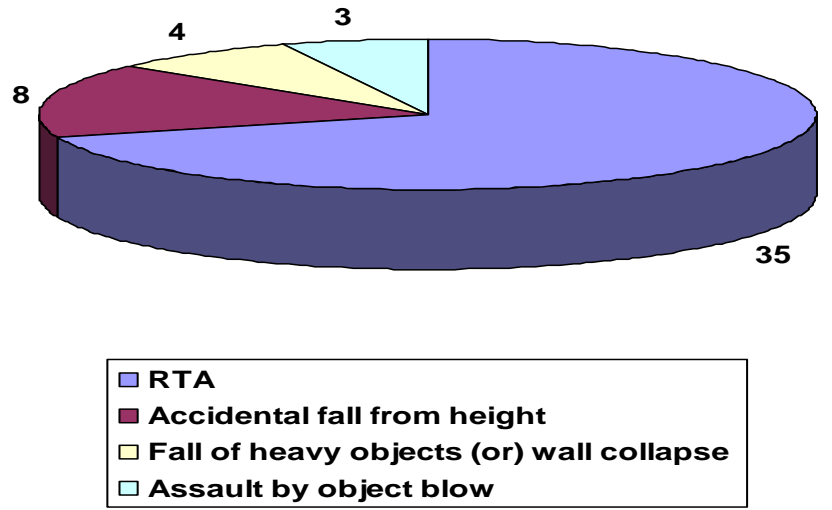

Male $-84 \%$, Female $-16 \%$ 


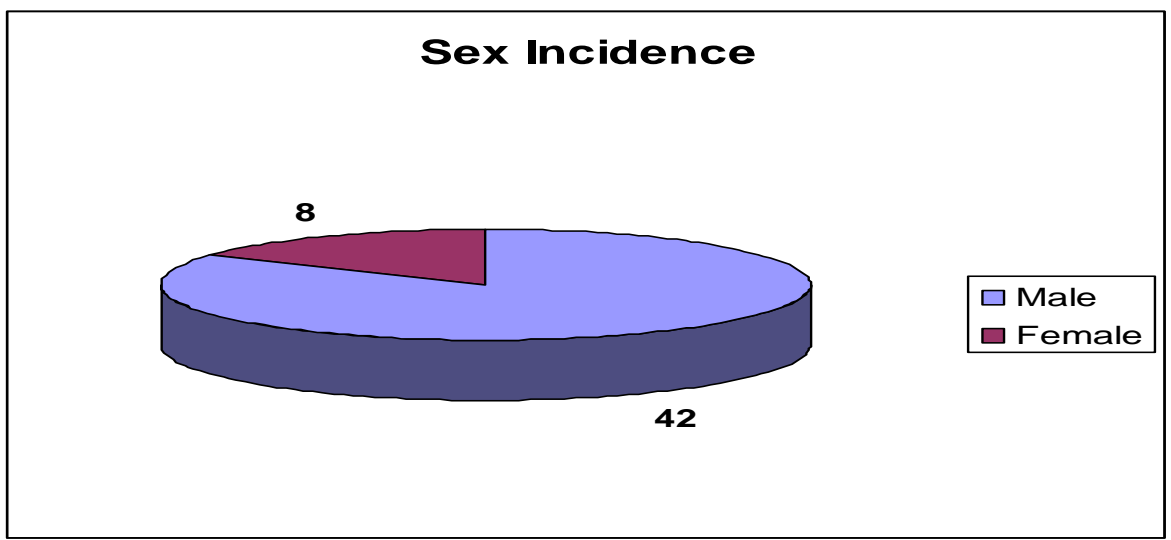

\begin{tabular}{|c|c|c|c|}
\hline & $>\quad$ Organ & $>$ & No. of cases \\
\hline$>$ & Stomach & $>$ & 3 \\
\hline$>$ & Jejunum & $>$ & 3 \\
\hline$>$ & Ileum & $>$ & 8 \\
\hline$>$ & Spleen & $>$ & 8 \\
\hline$>$ & Liver & $>$ & 8 \\
\hline$>$ & Pancreas & $>$ & 1 \\
\hline$>$ & Mesentry & $>$ & 3 \\
\hline 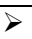 & Colon & $>$ & 3 \\
\hline$>$ & Rectum & $>$ & 1 \\
\hline 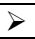 & Retroperitoneum & $>$ & 3 \\
\hline$>$ & Bladder & $>$ & 1 \\
\hline$>$ & Renal & $>$ & 3 \\
\hline
\end{tabular}

\section{Hollow Viscus}

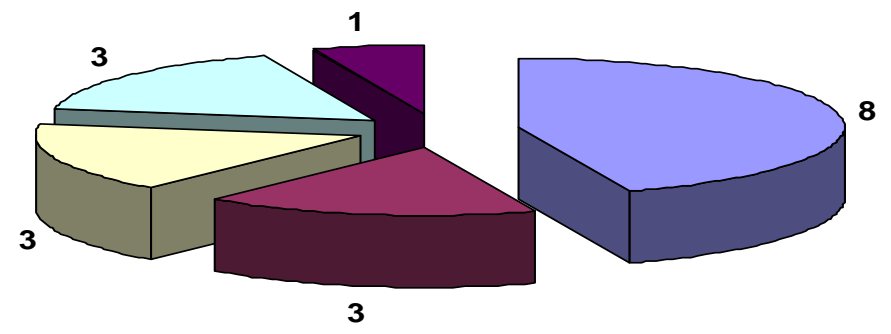

$\square$ lleum $\square$ Gastric $\square$ Jejunum $\square$ Colon $\square$ Rectum

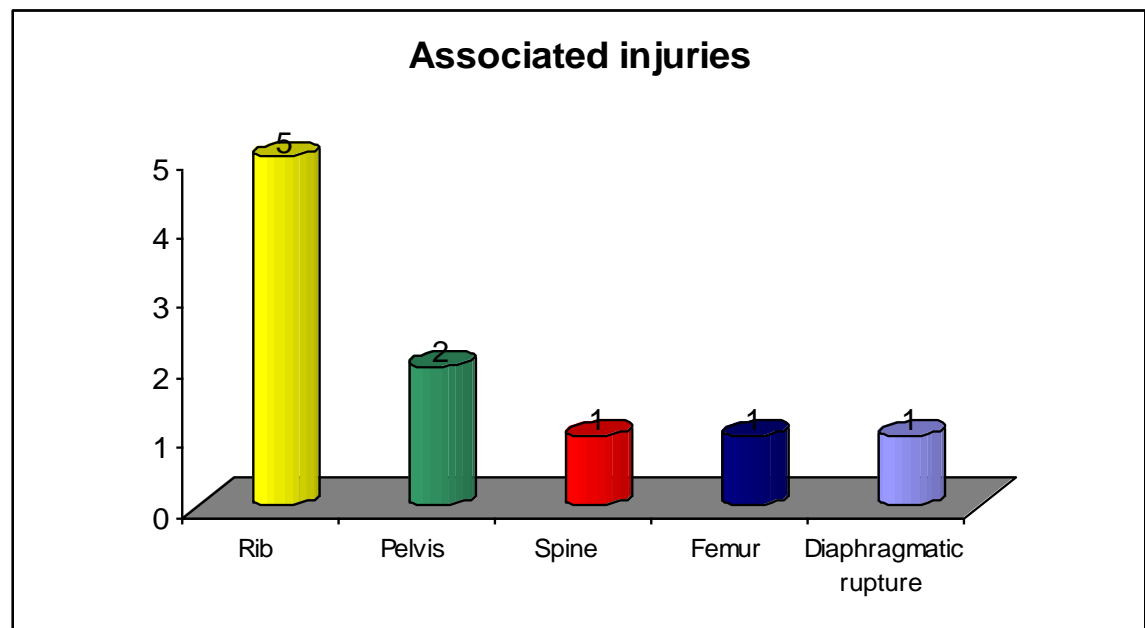




\section{Discussion}

In our study of blunt injury abdomen caused to road traffic accidents, accidental falls from height and assault by objects, observations were male in 50 cases admitted in Govt Rajaji Hospital, Madurai Medical College from December 2007 to October 2009. 5 cases were under 15 years of age and 38 cases were between the ages of 15 and 45 . Males accounts for 42 cases [ $84 \%$ ]. The proportionate majority of males could account for the male preponderance involved in vehicular traffic and outdoor activities, also to a certain extent by gender related aggressive driving behavior. Solid organ injuries like liver and splenic injuries due to direct compressive forces resulting from collision. Hollow viscus injuries might have been contributed by deceleration shear stress and sudden increase in intraabdominal pressure.

37 patients were admitted within 12 hours of the accident and the mortality in this group was one accounting for $2.7 \%$. Eleven patients came on the second day after having been treated in mofasul hospitals and the mortality in the group was one, calculated as $9 \%$, this case is a liver injury died postoperatively due to a combination of factors like protracted shock, septicemia and coagulopathy with multiple organ dysfunctions.

Two patients were admitted after 72 hours and the mortality in these 2 pts was 2 accounting for $100 \%$. Peritonitis and associated bony injuries were the cause of death in these patients who were not assessed and resuscitated adequately in the peripheral hospitals. Twenty patients [40\%] were hemodynamically unstable at presentation and required aggressive resuscitation. The Connecticut and saffolk studies show $44 \%$ shock incidence noted at the time of presentation. Physical examination revealed abdominal bruises in 10 patients and abdominal pain in all the patients and distension in 18 patients. Guarding was noted in 38 patients Rigidity was noticed in 19 patients. Davis et al reported abdominal pain in $75 \%$ of the patients; rigidity and rebound tenderness in $25 \%$ and $45 \%$ were clinically silent cases.

Associated major injuries increase the mortality and morbidity. The most common associated injuries were limb injuries followed by chest and head injuries. Basic laboratory investigations were of limited value. Plain skiagrams of the chest and abdomen revealed pneumoperitoneum in 11 cases and ground glass appearance in 19 patients. Fracture of the $10^{\text {th }}$ and $11^{\text {th }}$ rib was seen in three cases of splenic injury. Peritoneal tap has an accuracy ranging from 50 to $90 \%$ as was reported in a university of Toronto study $80 \%$ of the patients had a positive tap finding corresponding with the laparotomy findings. In our series, 40 patients [80\%] had a positive peritoneal. Hollow viscus injury [36\%] was the commonest injury detected in our series followed by splenic [16\%] and hepatic injuries [16\%].

Retroperitoneal hematoma was seen in 2 cases, one of whom had either a CT scan or USG evaluation during the in hospital period. No associated injury was noted in any of these cases. Associated head injury was seen in 1 patient who recovered completely with conservative management for the same. Seven cases presented with blunt injury abdominal findings with hemodynamic stability were treated conservatively. Two of them had renal injuries, one had liver and the other had spleen with the rest had no imaging abnormalities.Splenic injuries amounted to $16 \%$ of our cases. Three of the patients were hemodynamically stable at presentation. High velocity RTA was recorded consistently in all cases except one which is an assault by heavy object blow. Cobgil et al have reported that $\mathrm{CT}$ scan has a high sensitivity and efficacy in detecting splenic trauma. splenic salvage should be attempted if the condition of the patient, age and the spleen allows operative repair. Splenic injuries in our series were treated primarily by splenectomy except one case which is treated conservatively. Hepatic injuries there were 8 cases [16\% ]in our study and all of them gave history of direct trauma to all the right hypochondrium. Among these ten cases were found to involve the right lobe. One case was due to accidental fall from height. Shock was seen in 6 patients. Peritoneal tap revealed hemoperitoneum in 7 cases. One patient was admitted after 72 hours and he succumbed to death on the fourth postoperative day. Hepatoraphy was done in 6 cases, and one case was managed by packing. A case of hematoma right lobe of liver was managed conservatively with serial $\mathrm{CT}$ and frequent monitoring of vital parameters.

\section{Conclusion}

1. Blunt trauma to the abdomen in road traffic accidents, accidental fall from height and assault by object blow cause life threatening injuries in a significant number of cases.

2. The principal organs involved are in the order of incidence - hollow viscus, liver, spleen.

3. Males predominantly in the age group of $15-45$ are the most affected.

4. There is an increased incidence of complications and mortality when there is:

a. Inordinate delay in presentation and decision on treatment.

b. Inadequate pre-operative resuscitative measures.

c. Other unrelated risk factors for surgery.

d. The surgical procedure undertaken is unduly prolonged and complicated.

5. In the high risk patient who present with otherwise profound hypotension in spite of resuscitation it is safer to do laparotomy than to miss important injuries. In our study most of our cases were operated on the basis of 
physical findings and repeated clinical examination. This holds good for our country and other developing countries also.

- An attempt at splenic conservation is advocated especially in younger individuals.

- Low grade hepatic injuries in stable patients can be managed conservatively with serial evaluation through clinical and imaging modalities.

6. Since most of the cases were Medico-legal, the surgeon is biased towards an active surgical intervention without equivocating, so as not to miss even a minor injury or a masquerading majority injury.

7. The proper management of traumatic hollow viscus injuries requires the application of good clinical acumen, quick decisions, technique an adequate post-operative back up facilities and care. But, Technology is and always will be just a tool whereas qualified surgeons are irreplaceable.

\section{Bibliography}

[1]. Feliciano, David V.; Mattox, Kenneth L.; Moore, Ernest J (2012). Trauma, Seventh Edition (Trauma (Moore)). McGraw-Hill Professional. ISBN 978-0-07-166351-9.

[2]. Fitzgerald, J.E.F.; Larvin, Mike (2009). "Chapter 15: Management of Abdominal Trauma". In Baker, Qassim; Aldoori, Munther. Clinical Surgery: A Practical Guide. CRC Press. pp. 192-204. ISBN 9781444109627. 